\title{
Perfil epidemiológico e clínico de crianças vítimas de queimadura internadas em um centro de tratamento de queimados
}

\author{
Epidemiological and clinical profile of child burn victims admitted to a burn treatment center \\ Perfil epidemiológico y clínico de niños víctimas de quemaduras ingresados en un centro de \\ tratamiento de quemaduras
}

Recebido: 25/11/2021 | Revisado: 01/12/2021 | Aceito: 03/12/2021 | Publicado: 13/12/2021

Cíntia Helena Santuzzi

ORCID: https://orcid.org/0000-0002-3634-8602 Universidade Federal do Espírito Santo, Brasil E-mail: cintiasantuzzi@yahoo.com.br

Fernanda Mayrink Gonçalves Liberato ORCID: https://orcid.org/0000-0001-5227-2022 Universidade Federal do Espírito Santo, Brasil E-mail: nandamayrink@yahoo.com.br

Mariana Midori Sime

ORCID: https://orcid.org/0000-0001-6589-9692 Universidade Federal do Espírito Santo, Brasil E-mail: mariana.sime@ufes.br

Néville Ferreira Fachini de Oliveira ORCID: https://orcid.org/0000-0001-6958-8605 Universidade Federal do Espírito Santo, Brasil E-mail: neville.of@gmail.com

Rosalie Matuk Fuentes Torrelio ORCID: https://orcid.org/0000-0002-6159-4055 Hospital Infantil Nossa Senhora da Glória, Brasil E-mail: rosalie.plastica@gmail.com

Alysson Sgrancio do Nascimento ORCID: https://orcid.org/0000-0001-6535-1475 Universidade Federal do Espírito Santo, Brasil E-mail: alyssonsgrancio@gmail.com

Janaína de Alencar Nunes

ORCID: https://orcid.org/0000-0001-8561-5052 Universidade Federal do Espírito Santo, Brasil E-mail: janaina.nunes@ufes.br

Lucas Rodrigues Nascimento ORCID: https://orcid.org/0000-0002-6792-0819 Universidade Federal do Espírito Santo, Brasil

E-mail: prof.lucasnascimento@gmail.com

\begin{abstract}
Resumo
No Brasil não existe uma base de dados nacional que contemple o perfil dos pacientes vítimas de queimadura, portanto, o objetivo desse estudo foi descrever as características epidemiológicas, clínicas e da internação de crianças internadas em um centro de referência de tratamento de queimados (CTQ) do Espírito Santo. Foi realizado um estudo epidemiológico do tipo transversal e descritivo baseado em dados de prontuários eletrônicos e físicos das crianças internadas no CTQ no período de agosto de 2019 a julho de 2020. Dentro da amostra estudada, a maioria tem menos de cinco anos de idade, são do sexo masculino e apresentam como principal causa etiológica o calor $(93,1 \%)$. Em média, 14\% (DP 11) de superfície corporal queimada. As regiões corporais mais acometidas foram: tronco (64,7\%), membros superiores $(55,9 \%)$ e cabeça e pescoço $(44,1 \%)$. A profundidade da maioria das queimaduras foi de segundo grau $(86,3 \%)$ e com gravidade classificada como médio queimado (48\%). Os pacientes permaneceram em média 23 dias internados (DP=27, mínimo 3 e máximo 115 dias), sendo que $91,2 \%$ realizou pelo menos um procedimento de desbridamento da ferida. Os resultados desse estudo, somado ao conhecimento específico de uma região (Espírito Santo), podem auxiliar na elaboração de estratégias a fim de aumentar a conscientização acerca das queimaduras e fornecer orientação aos pais e ao público em geral, por meio de programas educacionais e campanhas de prevenção. Palavras-chave: Queimadura; Perfil epidemiológico; Espírito Santo; Centro de tratamento de queimados.
\end{abstract}

\footnotetext{
Abstract

In Brazil there is no national database that includes the profile of burn patients, therefore, the aim of this study was to describe the epidemiological, clinical and hospitalization characteristics of children admitted to a reference center for
} 
the treatment of burns ( CTQ) of Espírito Santo. A cross-sectional descriptive epidemiological study was carried out based on data from electronic and physical medical records of children admitted to the CTQ from August 2019 to July 2020. Within the sample studied, most are under five years old of age, are male and have heat as the main etiological cause $(93.1 \%)$. On average, 14\% (SD 11) of body surface was burned. The most affected body regions were: trunk (64.7\%), upper limbs (55.9\%) and head and neck (44.1\%). The depth of most burns was second degree (86.3\%) and severity classified as medium burn (48\%). Patients remained in the hospital for an average of 23 days (SD=27, minimum 3 and maximum 115 days), and 91.2\% underwent at least one wound debridement procedure. The results of this study, added to the specific knowledge of a region (Espírito Santo), can help in the development of strategies to raise awareness about burns and provide guidance to parents and the general public, through educational programs and prevention campaigns.

Keywords: Burn; Epidemiological profile; Espírito Santo; Burn treatment center.

\section{Resumen}

En Brasil no existe una base de datos nacional que incluya el perfil de los pacientes quemados, por lo que el objetivo de este estudio fue describir las características epidemiológicas, clínicas y de hospitalización de los niños ingresados en un centro de referencia para el tratamiento de quemaduras (CTQ) de Espíritu Santose realizó un estudio epidemiológico descriptivo transversal a partir de datos de la historia clínica electrónica y física de los niños ingresados en el CTQ deAgosto de 2019 a julio de 2020. Dentro de la muestra estudiada, la mayoría son menores de cinco años, son varones y tienen el celo como principal causa etiológica (93,1\%). En promedio, se quemó el 14\% (DE 11) de la superficie corporal. Las regiones corporales más afectadas fueron: tronco (64,7\%), miembros superiores $(55,9 \%)$ y cabeza y cuello $(44,1 \%)$. La profundidad de la mayoría de las quemaduras fue de segundo grado $(86,3 \%)$ y la gravedad se clasificó como quemadura media (48\%). Los pacientes permanecieron en el hospital una media de 23 días (DE = 27, mínimo 3 y máximo 115 días), y el 91,2\% se sometió al menos a un procedimiento de desbridamiento de la herida. Los resultados de este estudio, sumados al conocimiento específico de una región (Espírito Santo), pueden ayudar en el desarrollo de estrategias para concientizar sobre las quemaduras y orientar a los padres y al público en general, a través de programas educativos y campañas de prevención.

Palabras clave: Quemar; Perfil epidemiológico; Espírito Santo; Centro de tratamiento de quemaduras.

\section{Introdução}

Acidentes e traumas são as principais causas de morbimortalidade pediátrica. As queimaduras são o quarto tipo de trauma mais comum no mundo e uma das principais causas de mortalidade e incapacidade nos países em desenvolvimento (Rigon et al., 2019). São definidas como uma lesão tecidual podendo ser causada tanto por traumas elétricos, químicos, térmicos ou radioativos, tendo sua gravidade estipulada de acordo com a porcentagem do tecido acometido, período de exposição e agente causal (Sanches et al., 2016). Os métodos de classificação de extensão e profundidade da lesão por queimadura são bem estabelecidos e tem por objetivo, além de definir a gravidade das lesões, direcionar intervenções e prognóstico adequados. Na população pediátrica, as queimaduras profundas são mais comuns, uma vez que a espessura da epiderme e derme é menor em relação aos adultos, sendo o paciente considerado um grande queimado quando mais que $10 \%$ da superfície corporal estiver afetada (Sheridan, 2018).

Estima-se que cerca de 1 milhão de brasileiros sofrem acidentes com queimaduras anualmente, destes aproximadamente 120 mil necessitam de assistência médica e 2 a 3\% irão a óbito de forma direta ou indireta (Morais; Daga; Prestes, 2017). Segundo a Sociedade Brasileira de Queimaduras (2021), durante o período de março a novembro de 2020 houve um aumento de internações por queimaduras, sem considerar os acidentes que não necessitaram de hospitalização, devido ao intenso uso de álcool em gel e álcool líquido $70 \%$ como medida de prevenção do coronavírus. No período de setembro de 2020 a agosto de 2021 foram mais de 9000 internações de crianças e adolescentes vítimas de queimaduras no Brasil, sendo a principal faixa etária afetada de 1 a 4 anos (DATASUS, 2021). A queimadura é uma afecção traumática apontada como um grave problema para a saúde pública brasileira, tanto pelo número de pacientes acometidos quanto pelo gasto gerado no seu procedimento e acompanhamento pós-internação, uma vez que desta podem ocorrer sequelas psicológicas e físicas, causando uma alta taxa de morbidade e incapacidades (Bezerra; Galvão; Conceição; Da Silva; Andrade, 2020).

De acordo com a Organização Mundial da Saúde (OMS), o risco de morte por queimaduras na infância é alto, com uma taxa global de 3,9 mortes por 100.000. (World Health Organization, 2018). Estudos epidemiológicos apontam que 
menores de 5 anos têm as maiores taxas de mortalidade por queimaduras, sendo a maior incidência entre o primeiro e segundo ano de vida (Hernández et al., 2020). Tal condição justifica-se porque é nesta idade que a criança começa a andar, adquirindo liberdade, porém ainda não possui noção do perigo, tornando-se uma vítima fácil dos acidentes. (Salas et al., 2015). Nessa faixa etária a queimadura ainda chama maior atenção uma vez que o tecido cicatricial não apresenta as mesmas características que o tecido saudável, podendo repercutir com deformidades e prejuízo da função à medida que a criança se desenvolve (Oosterwijk et al., 2019).

No Brasil, apesar de alguns estudos epidemiológicos (Nigro et al., 2019; De Padua et al., 2020), não existe uma base de dados nacional que contemple o perfil dos pacientes vítimas de queimadura. Nesse sentido, pesquisas epidemiológicas regionais apresentam relevância e servem de base para estudos mais aprofundados. Portanto, o objetivo desse estudo foi descrever as características epidemiológicas, clínicas e da internação de crianças internadas em um centro de referência de tratamento de queimados. As perguntas específicas do presente estudo são:

1. Quais são as características epidemiológicas, clínicas (da queimadura) e da internação das crianças vítima de queimadura que necessitam de internação?

2. Quais fatores (e.x., idade, agente causador da queimadura, local da queimadura, necessidade de procedimentos cirúrgicos, uso de sonda ou ventilação assistida e tempo de internação) estão associados ao percentual da área de superfície queimada e ao agente etiológico?

Os achados podem auxiliar na identificação das características de crianças vítimas de queimadura relacionada ao percentual de área queimada e, portanto, podem orientar a prática clínica, sugerindo elaboração de estratégias específicas na prevenção de queimaduras.

\section{Metodologia}

\section{Desenho do estudo}

Foi realizado um estudo epidemiológico do tipo transversal e descritivo baseado em dados de prontuários eletrônicos e físicos das crianças internadas em um Centro de tratamento de Queimados (CTQ).

\section{Coleta de dados}

Foram incluídas todas as crianças que necessitaram de internação no setor de queimaduras do hospital público de referência do Estado do Espírito Santo. A coleta de dados é referente ao período de agosto de 2019 a julho de 2020, por meio de busca ativa nos prontuários eletrônicos e físicos das crianças e adolescentes de 0-18 anos que permaneceram internadas no CTQ no período do estudo. Não foram adotados critérios de exclusão. O projeto foi aprovado pelo comitê de ética e pesquisa da Universidade Federal do Espírito Santo (CAAE: 13787419.9.0000.5060). A coleta das variáveis de interesse do estudo ocorreu durante o mesmo período do estudo e os dados eram atualizados semanalmente de acordo com a evolução da equipe multiprofissional do setor. Os dados coletados foram organizados nas seguintes seções: características dos pacientes (idade, sexo, raça e região de moradia), características da queimadura (agente etiológico, percentual da superfície queimada, região corporal acometida, profundidade da queimadura, gravidade da queimadura) e característica da internação (tempo de internação, tipo de procedimento cirúrgico realizado, necessidade de uso de sonda vesical ou suporte ventilatório e ocorrência de morte).

\section{Características associadas ao percentual da superfície queimada e agente causador}

A variável dependente foi o percentual da superfície queimada e agente causador. As variáveis independentes foram organizadas em três grupos: 
- Características epidemiológicas: Idade, variável contínua em anos; Sexo, variável categórica nominal dicotômica: masculino e feminino; e Raça, variável categórica politômica: (1) negra, (2) parda e (3) branca.

- Características da queimadura: Agente etiológico, variável categórica dicotômica: (1) Térmica, (2) Elétrica e (3) Química; Profundidade da queimadura, variável categórica politômica: : (1) Segundo Grau e (2) Terceiro Grau; Gravidade da queimadura, variável categórica politômica: (1) Pequeno queimado, (2) Médio queimado e (3) Grande queimado e região corporal acometida, variável categórica dicotômica: (1) Cabeça e pescoço, (2) Tronco, (3) Membros Superiores, (4) Membros Inferiores e (5) Períneo,

- Características da Internação: Tempo de Internação, variável contínua em dias; Tipo de procedimento cirúrgico, variável categórica dicotômica: (1) desbridamento, (2) enxertia e (3) escarotomia; Necessidade de uso de sonda vesical ou suporte ventilatório, variável categórica dicotômica: sim ou não e ocorrência de morte, variável categórica dicotômica: sim ou não.

\section{Análise estatística}

A análise estatística dos dados foi realizada utilizando o Microsoft Office Excel 2013 e o Statistical Package for the Social Sciences (SPSS) versão 26.0. As características epidemiológicas, clínicas (da queimadura) e da internação foram apresentadas de forma descritiva. Variáveis categóricas foram expressas em frequências absolutas e relativas, e variáveis contínuas foram expressas em mediana (mínimo e máximo) ou média (desvio-padrão).

Teste Chi-quadrado, exato de Fisher ou correlação de Pearson foi utilizado para investigar associações entre o percentual da superfície queimada com as características epidemiológicas, da queimadura e da internação. A magnitude das correlações foi classificada como insuficiente $(\mathrm{r}<0.30)$, fraca $(0.30<\mathrm{r}<0.50)$, moderada $(0.50<\mathrm{r}<0.70)$ ou forte $(>0.70)$ (Mukaka, 2012).

\section{Resultados}

\section{Características epidemiológicas}

Durante um ano de coleta de dados 102 crianças foram internadas no CTQ. Dessas, 60,8\% eram do sexo masculino e com média de idade de 5 anos (DP 4), sendo que a maioria dos participantes eram pardos $(70,6 \%)$ e residiam na região metropolitana do estado do Espírito Santo 74,5\%. As características epidemiológicas estão descritas na Tabela 1. 
Tabela 1. Características das Crianças.

\begin{tabular}{|c|c|}
\hline Características & Crianças $(n=102)$ \\
\hline $\begin{array}{l}\text { Características Epidemiológicas } \\
\text { Idade (anos), média (DP) }\end{array}$ & $5(4)$ \\
\hline Sexo, número de meninos (\%) & $62(60,8)$ \\
\hline $\operatorname{Raça}(\%)$ & \\
\hline Pardo & $72(70,6)$ \\
\hline Preto & $15(14,7)$ \\
\hline Branco & $15(14,7)$ \\
\hline Região onde Mora (\%) & \\
\hline Metropolitana & $76(74,5)$ \\
\hline Central & $14(13,7)$ \\
\hline Norte & $8(7,8)$ \\
\hline Sul & $4(3,9)$ \\
\hline Características Clínicas da queimadura & \\
\hline Agente Etiológico (\%) & \\
\hline Térmico & $95(93,1)$ \\
\hline Químico & $5(4,9)$ \\
\hline Elétrico & $2(2)$ \\
\hline Profundidade da queimadura (\%) & \\
\hline Segundo Grau & $88(86,3)$ \\
\hline Terceiro Grau & $14(13,7)$ \\
\hline Gravidade da queimadura (\%) & \\
\hline Pequeno Queimado & $22(21,6)$ \\
\hline Médio Queimado & $49(48)$ \\
\hline Grande queimado & $31(30,4)$ \\
\hline Região corporal acometida $(\%)$ & \\
\hline Cabeça e pescoço & $45(44,1)$ \\
\hline Tronco & $66(64,7)$ \\
\hline Membros Superiores & $57(55,9)$ \\
\hline Membros Inferiores & $36(35,3)$ \\
\hline Períneo & $3(2,9)$ \\
\hline $\begin{array}{l}\text { Percentual da Superfície Queimada (\%), } \\
\text { média (DP) }\end{array}$ & $14(11)$ \\
\hline Características da Internação & \\
\hline Tempo de Internação (dias), média (DP) & $23(27)$ \\
\hline Tipo de procedimento cirúrgico e uso de & \\
\hline Sonda/Suporte Ventilatório (\%) & $93(91,2)$ \\
\hline Desbridamento & $24(23,5)$ \\
\hline Enxertia & $3(2,9)$ \\
\hline Escarotomia & $9(8,8)$ \\
\hline Uso de Sonda Vesical & $6(5,9)$ \\
\hline Uso de Suporte Ventilatório & \\
\hline Ocorrência de Morte (\%) & $1 \%$ \\
\hline
\end{tabular}

DP = Desvio padrão. Fonte: Prontuários eletrônicos e físicos do Centro de Tratamento de Queimados do Espírito Santo.

\section{Características Clínicas da Queimadura}

O principal agente etiológico foi queimadura térmica (93,1\%). Em média, 14\% (DP 11) de percentual da superfície queimada. As regiões corporais mais acometidas foram: queimadura de tronco em $64,7 \%$ dos casos, seguido por membros superiores $(55,9 \%)$ e cabeça e pescoço $(44,1 \%)$. As regiões menos acometidas foram membros inferiores $(35,3 \%)$ e região de 
períneo $(2,9 \%)$. A profundidade da maioria das queimaduras foi de segundo grau $(86,3 \%)$ e com gravidade classificada como médio queimado (48\%). Esses dados estão resumidos na Tabela 1.

\section{Características da internação}

Os pacientes permaneceram em média 23 dias internados ( $\mathrm{DP}=27$, mínimo 3 máximo 115 dias) no centro de tratamento de queimados. Sendo que $91,2 \%$ realizou pelo menos um procedimento de desbridamento da ferida, $23,5 \%$ necessitou de enxertia e 2,9\% realizou escarotomia. Somente 5,9\% dos pacientes necessitaram de suporte ventilatório e 8,8\% dos pacientes usaram sonda vesical. A ocorrência de morte foi de 1\%. Esses dados estão resumidos na Tabela 1.

\section{Características epidemiológicas, clínica e da internação associadas ao percentual da área queimada e ao agente etiológico.}

A Tabela 2 apresenta o coeficiente de correlação entre as características das crianças e o percentual da área queimada. Dentre as características epidemiológicas avaliadas, a idade $(r=0.2 ; p=0.01)$ apresentou uma correlação positiva insuficiente com o percentual da área queimada e uma associação com o agente etiológico $\left(x^{2}=44,9 ; p=0,007\right)$.

Para as características clínicas houve associação entre as regiões do corpo queimada: cabeça, pescoço, face $\left(x^{2}=9,2\right.$; $p=0.002)$ e tronco $\left(x^{2}=13,0 ; p=0.001\right)$ com o percentual da área queimada.

As características da internação apresentaram associação entre o percentual da área queimada e a necessidade de enxertia $\left(x^{2}=15,9 ; p<0.001\right)$ e uso de sonda vesical $\left(x^{2}=5,5 ; p=0.032\right)$, e uma correlação positiva e fraca com o tempo de internação $(r=0.40 ; p<0.001)$.

Tabela 2. Associação entre as características das crianças e o percentual da área queimada e agente etiológico. (n=102).

\begin{tabular}{lcc}
\hline \multicolumn{1}{c}{ Características } & $\begin{array}{c}\text { Percentual de área } \\
\text { queimada }\end{array}$ & Agente Etiológico \\
\hline Características Epidemiológicas & $r=0,25 ; \boldsymbol{p}=\mathbf{0 , 0 1}$ & $x^{2}=44,9 ; \boldsymbol{p}=\mathbf{0 , 0 0 7}$ \\
$\quad$ Idade & $x^{2}=33,4 ; p=0,80$ & $x^{2}=1,70 ; p=0,50$ \\
$\quad$ Sexo & $x^{2}=33,4 ; p=0,80$ & $x^{2}=3,10 ; p=0,58$ \\
$\quad$ Raça & & \\
Características Clínicas da & & \\
Queimadura & $x^{2}=9,24 ; \boldsymbol{p}=\mathbf{0 , 0 0 2}$ & $x^{2}=0,90 ; p=0,83$ \\
$\quad$ Cabeça e pescoço & $x^{2}=13,0 ; \boldsymbol{p}=\mathbf{0 , 0 0 1}$ & $x^{2}=3,50 ; p=0,13$ \\
Tronco & $x^{2}=0,30 ; p=0,58$ & $x^{2}=1,68 ; p=1,00$ \\
Membros superiores & $x^{2}=0,90 ; p=0,34$ & $x^{2}=1,36 ; p=0,69$ \\
Membros inferiores & $x^{2}=2,85 ; p=0,24$ & $x^{2}=1,18 ; p=0,55$ \\
Períneo & $x^{2}=132,9 ; p=0,07$ & - \\
Agente etiológico & & $x^{2}=132,9 ; p=0,07$ \\
Percentual da área queimada & $r=0,42 ; \boldsymbol{p}=\mathbf{0 , 0 0 1}$ & $x^{2}=128,95 ; p=0,35$ \\
Características da Internação & $x^{2}=3,5 ; p=0,08$ & $x^{2}=1,78 ; p=0,49$ \\
Dias de Internação & $x^{2}=15,9 ; \boldsymbol{p}=\mathbf{0 , 0 0 1}$ & $x^{2}=2,35 ; p=0,22$ \\
Desbridamento & $x^{2}=2,86 ; p=0,24$ & $x^{2}=1,64 ; p=1,00$ \\
Enxertia & $x^{2}=5,53 ; \boldsymbol{p}=\mathbf{0 , 0 0 3}$ & $x^{2}=5,15 ; p=0,12$ \\
Escarotomia & $x^{2}=2,51 ; p=0,21$ & $x^{2}=2,95 ; p=0,35$ \\
Sonda Vesical & & \\
Suporte Ventilatório & &
\end{tabular}

$r=$ Pearson correlation coefficient; $x^{2}=$ Chi-square/ Exato de Fisher. Fonte: Prontuários eletrônicos e físicos do Centro de Tratamento de Queimados do Espírito Santo.

\section{Discussão}

O estudo objetivou identificar as características epidemiológicas e clínicas de crianças que necessitaram de internação devido à queimadura. No geral, o perfil encontrado foi de meninos com média de 5 anos de idade residentes na região metropolitana do estado do Espírito Santo, sendo o agente etiológico, em 93\% dos casos, de origem térmica, com a principal 
área queimada o tronco com queimaduras de segundo grau e gravidade classificada como médio queimado e com percentual de área corporal queimada em média de 14\%. Quanto às características da internação, as crianças permaneceram, em média, 23 dias internadas e mais de $90 \%$ necessitaram de procedimento cirúrgico do tipo desbridamento. Foi observada uma correlação positiva e fraca entre a idade e tempo de internação com o percentual da área queimada e houve uma associação entre as regiões de cabeça/pescoço e tronco, necessidade de enxertia e uso de sonda vesical com o percentual da área queimada e o agente etiológico associou-se a idade.

Os resultados desse estudo indicam que em relação às características epidemiológicas das crianças internadas eram mais prevalentes crianças do sexo masculino com média de idade de 5 anos. Corroborando com nossos dados, o sexo masculino apresenta uma frequência maior nos trabalhos sobre queimaduras (Amador et al., 2021 \& Correia et al., 2019), tais como estudo de Iqbal e Saaiq (2011), que avaliou um total de 1725 crianças vítimas de queimaduras das quais $67 \%$ eram do sexo masculino, taxa semelhante ao nosso estudo $(60,8 \%)$. No mesmo sentido, muitos estudos epidemiológicos descrevem que crianças com faixa etária menor de 5 anos são as mais suscetíveis a acidentes por queimadura (Correia et al., 2019; Iqbal \& Saaiq, 2011; Dassie \& Alves, 2011). Isso é explicado pelo grau de exposição em brincadeiras de risco e ainda pelo comportamento diferente entre gênero, assim como pelos fatores culturais, que propiciam aos meninos mais liberdade, em comparação às meninas (Francisconi et al., 2016). Além disso, fatores que podem explicar a maior prevalência de acidentes em menores de 5 anos estão relacionadas às características do desenvolvimento infantil, visto que as crianças são imaturas e curiosas em relação ao meio ambiente, estando, portanto, mais expostas a situações de perigo, que é potencializado por monitoramento inadequado (Martins \& AndradE, 2007).

Queimaduras térmicas são as lesões provocadas por fontes de calor como o fogo, líquidos ferventes, vapores, objetos quentes ou exposição ao sol (Tolles, 2018). Nossos resultados indicam que 93,1\% das crianças sofreram queimadura por este agente etiológico, estando em consonância com muitos estudos em todo o mundo, os quais demonstraram que as lesões térmicas são mais comuns em crianças menores de 5 anos de idade (Alnababtah et al., 2016). Ademais et al. (2015), afirmam que crianças apresentam mais comumente queimaduras por escaldadura, ao passo que as queimaduras por fogo são vistas com mais frequência em adolescentes, em consonância com esses dados, nosso estudo aponta uma associação entre o agente etiológico e idade da criança. É importante considerar que, a avaliação de um paciente com lesão térmica inclui análise do tamanho, profundidade e localização da queimadura. A população avaliada nesse estudo apresentou queimaduras de segundo grau, com classificação de médio queimada, entretanto, com média de 14\% de superfície corporal queimada, o que considerase um alto valor, uma vez que, para crianças, superfícies corporais queimadas acima de $10 \%$ já são consideradas grandes queimaduras (Partain et al., 2020). Nossos achados ainda apontam que quanto maior o percentual de área queimada maior a idade da criança e mais dias de internação serão necessárias, além de uma associação entre queimaduras em cabeça/pescoço e tronco e percentual de área queimada. Esses achados podem ser confirmados ao avaliarmos o método de cálculo para estimar área queimada (Gomes et al., 1997), o qual, para crianças estima-se uma área maior nas regiões onde foram encontradas associações (cabeça e tronco).

Quanto às características da internação, nossa amostra demonstrou um tempo médio de permanência hospitalar de 23 dias e ocorrência de morte de 1\%. Em outros estudos em CTQs no Brasil descrevem tempo de internação entre 10 - 16 dias (Dassie \& Alves, 2011; Nigro et al., 2009; Biscegli et al., 2014), demonstrando que nossa amostra apresentou um período elevado de internação. Sabe-se que a permanência prolongada em internações apresenta consequências para a saúde física e emocional do indivíduo, aumentando risco de morbidade, desnutrição, depressão, estados confusionais, infecções, complicações iatrogênicas, diminuição da mobilidade e maior nível de dependência (Silva et al., 2018). Para crianças esse cenário parece ser agravado, pois as crianças que experimentam hospitalização prolongada encontram-se em maior risco de retardo de desenvolvimento neuropsicomotor (Ferreira et al., 2020), além disso, alguns autores descrevem os prejuízos 
emocionais com consequências na vida adulta (Gomes \& Nobrega, 2015). Por outro lado, a ocorrência de morte encontrada parece estar em consonância com outros estudos brasileiros (Dassie \& Alves, 2011; Nigro et al., 2009). Nossos resultados sugerem ainda que quanto maior o percentual de área queimada maior o tempo de internação e uma associação entre necessidade de enxertia e uso de sonda vesical com percentual de área queimada, o que parece estar relacionado com a população estudada que apresenta uma maior porcentagem de área queimada e maior tempo de internação comparado com outros estudos em crianças queimadas.

Esse estudo não está livre de limitações, sendo que a principal está no fato de o trabalho ser baseado em dados secundários de registro de prontuários, os quais, por muitas vezes observa-se dificuldade como: a falta de padronização de registros, registros incompletos e/ou ilegíveis e até dificuldade em localizar e acessar os registros. Entretanto, o Conselho Federal de Medicina orienta que os registros em prontuários sejam completos, coerentes, legíveis e contenham informações acerca das condições do paciente, além disso, é proposto que os prontuários possuam caráter sigiloso, legal e científico (Conselho Federal De Medicina, 2002).

\section{Conclusão}

Dentro da amostra estudada, as crianças vítimas de queimadura que necessitam de internação no estado do Espírito Santo têm em sua maioria menos de cinco anos de idade, são do sexo masculino e apresentam como principal causa etiológicade origem térmica. Os resultados desse estudo, somado ao conhecimento específico de uma região (Espírito Santo), podem auxiliar na elaboração de estratégias a fim de aumentar a conscientização acerca das queimaduras e fornecer orientação aos pais e ao público em geral, por meio de programas educacionais e campanhas de prevenção. Ademais, não somente o impacto físico deve ser considerado, mas também o aspecto emocional e consequências psicológicas, uma vez que verificou-se um alto índice de tempo de internação em nossa amostra. As consequências das queimaduras, em especial na população pediátrica, devem ser devidamente abordadas e divulgadas a fim de minimizar as sequelas. Neste processo, todos os atores sociais (família, escola, profissionais da saúde e governantes) devem desempenhar um papel importante na promoção e prevenção de acidentes por queimaduras. Sugerimos que estudos futuros explorem as características das diferentes regiões brasileiras a fim de compreender o perfil das crianças internadas no Brasil.

\section{Agradecimentos}

À Universidade Federal do Espírito Santo, a secretaria de Estado da Saúde - SESA e ao Hospital Infantil Nossa Senhora da Glória - HEINSG pela participação do projeto de pesquisa do Programa Institucional de Bolsas de Iniciação Científica (Bolsa UFES) e a todos os colegas que contribuíram neste trabalho.

\section{Referências}

Alnababtah, K., Khan, S., \& Ashford, R. (2016). Socio-demographic factors and the prevalence of burns in children: an overview of the literature. Paediatrics and International Child Health, 36 (1), 45-51. https://doi.org/10.1179/2046905514Y.0000000157

Amador, A. V. C., Mazarakis, L. P. G., \& Felzemburgh, V. A. (2021). Perfil dos pacientes em Unidade de Terapia Intensiva de queimados em hospital de referência. J. of Multiprofessional Health Research, 2 (1), 52-71.

Bezerra, A. F. C., Galvão, P. V. M., Conceição, J. M., da Silva, J. M. N., \& Andrade, E. S. S. (2020). Mortality to burns in children between zero and four years in Brazil. Braz. J. of Develop, 6 (7), 43012-43023. https://doi.org/ 10.34117/bjdv6n7-062

Biscegli, T. S., Benati, L. D., Faria, R. S., Boeira, T. R., Cid, F. B., \& Gonsaga, R. A. T. (2014). Profile of children and adolescents admitted to a Burn Care Unit in the countryside of the state of São Paulo. Rev Paul Pediatr, 32 (3), 177-182. https://doi.org/10.1590/0103-0582201432305

Conselho Federal de Medicina (Brasil). Resolução n ${ }^{\circ}$. 1.638, de 10 de julho de 2002. Define prontuário médico e torna obrigatória a criação da Comissão de Revisão de Prontuários nas instituições de saúde. Diário Oficial da União 9 ago 2002 , Seção 1. http://www.rio.rj.gov.br/dlstatic/10112/5125745/4209117/RESOLUCAOCFMN1.638DE10DEJULHODE2002.pdf 
Correia, D. S., Chagas, R. R. S., Costa, J. G., Oliveira, J. R., França, N. P. A., \& Taveira, M. G. M. M. (2019). Perfil de crianças e adolescentes internados no centro de terapia de queimados. Rev enferm UFPE, 13 (5), 1361-1369. https://doi.org/10.5205/1981-8963-v13i5a239133p1361-1369-2019

DATASUS (2021). Morbidade hospitalar do SUS. http://tabnet.datasus.gov.br/cgi/deftohtm.exe?sih/cnv/nruf.def.

Dassie, L. T. \& Alves, E. O. (2011). Centro de tratamento de queimados: perfil epidemiológico de crianças internadas em um hospital escola. Rev Bras Queimaduras, $10(1), 10-14$

De Padua, G. A. C., Nascimento, J. M., Quadrado, A. L. D., Perrone, R. P., \& Junior, S. C. S. (2017). Epidemiology of burn cases hospitalized at the Plastic Surgery and Burns Service of Santa Casa de Misericórdia de Santos, Brazil. Rev. Bras. Cir. Plást, 32 (4), 550-555. http://www.dx.doi.org/10.5935/21771235.2017RBCP0089

Ferreira, A. N., de Sales, J. K. D., Coelho, H. P., Marçala, F. A., de Melo, C. S., de Sousa, D. R., \& Feitosa, A. C. (2020). Hospitalização infantil: impacto emocional indexado a figura dos pais. Revista Interfaces, 8 (1), 402-408.

Francisconi, M. H. G., Itakussu, E. Y., Valenciano, P. J., Fujisawa, D. S., \& Trelha, C. S. (2016). Epidemiological profile of children with burns admitted in a Burn Unit. Rev Bras Queimaduras, 15 (3), 137-141.

Gomes, D. R., Serra, M. C., \& Pellon, M. A. (1997). Tratamento de Queimaduras: um guia prático. Revinter.

Gomes, G. L. L. \& Nobrega, M. M. L. (2015). Ansiedade da hospitalização em crianças: proposta de um diagnóstico de enfermagem. Rev. Latino-Am, 23 (5), 963-970. https://doi.org/10.1590/0104-1169.0372.2637

Gonzalez, R. \& Shanti, C. M. (2015). Overview of current pediatric burn care. Seminars in Pediatric Surgery, 24 (1), 47-49. https://doi.org/10.1053/j.sempedsurg.2014.11.008

Hernández, C. M. C., Núñez, V. P., Suárez, F. A. P., Banqueris, R. F., García, M. S., \& Mendoza, D. P. (2020). Burns and prevention in children. Rev Bras Queimaduras, 19 (1), 84-88.

Iqbal, T. \& Saaiq, M. (2011). The burnt child: an epidemiological profile and outcome. J Coll Physicians Surg Pak, 21 (11), 691-694.

Malta, D. C., Berna, R. T. I., de Lima, C. M., Cardoso, L. S. M., de Andrade, F. M. D., Marcatto, J. O., \& Gawryszewski, V. P. (2020). Perfil dos casos de queimadura atendidos em serviços hospitalares de urgência e emergência nas capitais brasileiras em 2017. Rev Bras Epidemiol, 23 (1), 1-14. https://doi.org/10.1590/1980-549720200005.supl.1

Martins, C. B. \& Andrade, S. M. (2007). Queimaduras em crianças e adolescentes: análise da morbidade hospitalar e mortalidade. Acta Paul Enferm, 20 (4), 464-469. https://doi.org/10.1590/S0103-21002007000400013

Morais, I. H., Daga, H., \& Prestes, M. A. (2017). Crianças queimadas atendidas no Hospital Universitário Evangélico de Curitiba: perfil epidemiológico. Rev Bras Queimaduras, 15 (4), 256-260.

Mukaka, M. M. (2012). Statistics corner: A guide to appropriate use of correlation coefficient in medical research. Malawi Med J, 24 (3), 69-71.

Nigro, M. V. A. S., Maschietto, S. M., Damin, R., Costa, C. S., \& Lobo, G. L. A. (2019). Perfil epidemiológico de crianças de 0-18 anos vítimas de queimaduras atendidas no Serviço de Cirurgia Plástica e Queimados de um Hospital Universitário no Sul do Brasil. Rev Bras Cir Plást, 34 (4), 504-508. http://www.dx.doi.org/10.5935/2177-1235.2019RBCP0230

Nigro, M. V., Freitas, E. T., Junior, S. C. L., Dalcumune, F., Netto, R. F. B,, Sanches, M. E., \& Piper, P. (2009). Perfil epidemiológico das crianças internadas por queimadura no Hospital Universitário Evangélico de Curitiba (HUEC) no período de julho de 2007 a fevereiro de 2008. Arq Catar Med, 38 (1), $172-174$.

Oosterwijk, A. M., Mouton, L. J., Akkerman, M., Stoop, M. M., Van Baar, M. E., Scholten-Jaegers, S. M. H., Van der Schans, C. P., \& Nieuwenhuis, M. K. (2019). Course of prevalence of scar contractures limiting function: A preliminary study in children and adolescents after burns. Burns. 45 (8), 1810-1818. https://doi.org/10.1016/j.burns.2019.05.003

Partain, K. P., Fabia, R., \& Thakkar, R. K. (2020). Pediatric burn care: new techniques and outcomes. Current Opinion in Pediatrics, 32 (3), 405-410. https://doi.org/10.1097/MOP.0000000000000902

Rigon, A. P. Gomes, K. K. Posser, T. Franco, J. L. Knihs, P. R., \& de Souza, P. A. (2019). Perfil epidemiológico das crianças vítimas de queimaduras em um hospital infantil da Serra Catarinense. Rev Bras Queimaduras, 18 (2), 107-112.

Salas, A., Muñoz, I., Sierra, M., Merchán Galvis, A. M., Castro, O., \& Bonilla, J. (2015). Quemaduras en menores de cinco años en Popayán, Colombia: creencias, conocimientos y prácticas. Pediatría, 48 (1), 21-26. https://doi.org/10.1016/j.rcpe.2015.07.003

Sanches, P. H. S., Sanches, J. A., Nogueira, M. J., Perondi, N. M., Sugai, M. H., Justulin, A. F., Vantine, G. R., \& Neto, O. T. (2016). Perfil epidemiológico de crianças atendidas em uma Unidade de Tratamento de Queimados no interior de São Paulo. Rev Bras Queimaduras, 15 (4), $246-250$.

Sheridan, R. L. (2018). Burn Care for Children. Pediatrics in Review, 39 (6), 273-286. https://doi.org/10.1542/pir.2016-0179

Silva, R. P., Pinto, P. I., \& Alencar, A. M. C. (2018). Efeitos da hospitalização prolongada: o impacto da internação na vida do paciente e seus cuidadores. Revista Saúde, 44 (3), 1-12. https://doi.org/10.5902/2236583424876

Sociedade Brasileira de Queimaduras (2021). Álcool: De aliado a vilão na pandemia. https://sbqueimaduras.org.br/noticia/alcool-de-aliado-a-vilao-napandemia-.

Tolles, J. (2018). Emergency department management of patients with thermal burns. Emerg Med Pract, 20 (2), 1-24.

World Health Organization (2018). Burns. https://www.who.int/news-room/fact-sheets/detail/burns. 